\title{
ARBOLADO DE ESPACIOS VERDES DE LA CIUDAD DE CORRIENTES: RELEVAMIENTO DE PLAGAS Y ENFERME- DADES, ASESORAMIENTO Y CAPACITACIÓN TÉCNICA.
}

Laffont, E. R. ${ }^{1}$; M. C. Godoy ${ }^{1}$; E. Galdeano ${ }^{2}$; J. M. Coronel ${ }^{1}$ y C. Etcheverry ${ }^{1}$

\section{RESUMEN}

El arbolado urbano presta importantes servicios socio- ambientales y forma parte del patrimonio cultural e histórico. El estrés a que están sometidos los árboles urbanos los predispone al ataque de diversas plagas y enfermedades. En la ciudad de Corrientes (Argentina) se desarrolló un proyecto de extensión destinado a analizar la situación sanitaria de los árboles de espacios verdes de la ciudad y colaborar en la capacitación del personal municipal afectado a su atención. Se analizaron un total de 779 árboles pertenecientes a 67 especies y los porcentajes de árboles afectados variaron entre el $27,59 \%$ y el $20,91 \%$. Los principales agentes perjudiciales resultaron los hongos de la madera, hormigas, termitas y pulgones. Se elaboraron e implementaron de medidas de manejo y prevención en aquellos árboles con mayores daños. Se realizó un curso- taller teórico-práctico destinado a funcionarios y agentes municipales del área, referido a la importancia ambiental del arbolado urbano, la identificación de los principales causantes de daños en $\mathrm{Co}^{-}$ rrientes y sus posibles medidas de manejo.

\section{ABSTRACT}

The urban trees provide important social and environmental services and participate of the cultural and historical heritage. Those trees are subjected to stress that predisposes them to the attack by various pests and diseases. In the city of Corrientes (Argentina), an extension project aimed at analyzing the health status of trees in green spaces and assist in the training of the city staff was developed. A total of 779 trees of 67 species were analyzed. The percentages of affected trees varied between $27.59 \%$ and $20.91 \%$. Main harmful agents were wood fungi, ants, termites and aphids. Management and prevention measures were developed and implemented in those trees with major damage. One theoretical and practical workshop for municipal officials and agents of the area was carried out, focusing on the environmental importance of urban trees and identifying the main causes of damage in Corrientes and its management.

\footnotetext{
${ }^{1}$ - Laboratorio de Biología de los Invertebrados. Facultad de Ciencias Exactas y Naturales y Agrimensura (Universidad Nacional del Nordeste). Av. Libertad 5470. Corrientes, Argentina. erl@exa.unne.edu.ar

${ }^{2}$ - Laboratorio de Fisiología. IBONE (CONICET_UNNE). Facultad de Ciencias Agrarias (UNNE). Sargento Cabral 2131. Corrientes, Argentina.
} 
PALABRAS CLAVE: Arbolado urbano, plagas, enfermedades, monitoreo, $\mathrm{Co}^{-}$ rrientes

\section{INTRODUCCIÓN}

El medio urbano es un ecosistema con alto grado de disturbios y alteraciones de origen antrópico. Por ello, uno de los mayores desafíos de su gestión es tratar de mantener en las ciudades un ambiente sano y orientado a la obtención de una mejor calidad de vida. Precisamente, entre los factores de calidad de vida de sus habitantes se consideran los espacios verdes urbanos (Gómez Lopera 2005).

Los parques, plazas y paseos de las ciudades, además de ser espacios útiles para el descanso y la recreación, constituyen sitios idóneos para la educación ambiental y la promoción del cuidado de la naturaleza. Los árboles situados en estos espacios verdes prestan servicios socio- ambientales fundamentales, ya que definen la fisonomía local y poseen un efecto protector y regulador del ambiente urbano, actuando principalmente como elementos estabilizadores micro- climáticos. Contribuyen además a la disminución de la contaminación atmosférica por los procesos de liberación de oxígeno, retención de partículas y polvo en suspensión y amortiguación de ruidos, participando asimismo en la conservación de la diversidad biológica urbana. A estos beneficios se agregan aquellos aspectos subjetivos relacionados con el bienestar psicológico y anímico de los habitantes (Buduba 2004). En la ciudad de Corrientes, la antigüedad del casco histórico hace que muchos ejemplares arbóreos, además de las funciones antes mencionadas, formen parte del patrimo- nio cultural e histórico, como el caso de la tradicional higuera del género Ficus situada en el Parque Mitre, cuya edad sería de alrededor de un siglo, de acuerdo a las estimaciones que se realizan (Cian et al. 2000).

Los árboles urbanos se hallan sometidos a estrés constante por el ambiente artificial adverso y por el manejo a veces incorrecto que los predispone al ataque de diversas plagas y enfermedades. Entre las plagas animales, el grupo más importante es el de los insectos fitófagos y xilófagos, principalmente de los ordenes Coleoptera (taladros, picudos y escarabajos), Lepidoptera (orugas), Hymenoptera (hormigas cortadoras y forrajeras) e Isoptera (termitas) (Fiorentino \& Diodato 1991). Los géneros y especies de insectos involucrados varían de acuerdo a la especie vegetal y a la región biogeográfica. Otro aspecto importante a tener en cuenta es que, al menos en el caso de los isópteros y otros insectos xilófagos, los árboles actúan como reservorio de estas plagas que ocasionalmente causan infestaciones a viviendas cercanas (Fontes 1995, 1998; Milano \& Fontes 2002). Los daños causados por insectos, podas o la ruptura de ramas viejas debida a vientos fuertes, facilitan el ingreso de hongos xilófagos que pueden atacar tanto el tronco como las raíces de los árboles (Comerci 2010). Entre otras enfermedades podemos mencionar al "declinamiento del paraíso", causado por un fitoplasma (procariota sin pared celular) que se ha difundido en muchas ciudades del país causando la muerte masiva de estos árboles (Galdeano et al. 2004).

Las estrategias de manejo pretenden conservar y valorizar el patrimonio ecológico y socio- cultural representado por los 
árboles de espacios verdes en los ambientes urbanos en general. La gestión del arbolado requiere evaluaciones periódicas, para la detección de plagas y enfermedades, así como de los daños producidos. Dicha tarea debe ser llevada a cabo por personal capacitado en esta temática y con experiencia profesional en los aspectos de diagnóstico y tratamiento de las afecciones mencionadas (Kopta et al. 1997).

A fin de conocer y analizar la situación del ataque de plagas y enfermedades al arbolado urbano en la ciudad de Corrientes y colaborar en la capacitación del personal municipal que trabaja en dicha área, se desarrolló el Proyecto de Extensión "Arbolado de espacios verdes de la Ciudad de Corrientes: relevamiento de plagas y enfermedades, asesoramiento y capacitación técnica" comprendido en el Programa UNNE en el Medio, en el que participaron estudiantes y docentes de las Facultades de Ciencias Exactas (Biología) y Ciencias Agrarias de la UNNE.

\section{MATERIALES Y MÉTODOS}

Las actividades se llevaron a cabo en tres etapas, a lo largo de un período de doce meses. En la primera etapa se realizaron relevamientos que comprendieron el total de árboles localizados en los siguientes espacios verdes del casco histórico de la ciudad de Corrientes: Parque Mitre, Parque Cambá Cuá, Plaza Torrent y Plaza de la Cruz. Los mismos estuvieron a cargo de los docentes y estudiantes participantes en el proyecto. Para cada ejemplar se realizó la determinación taxonómica y se registró su circunferencia. Se reconoció el estado sanitario de cada árbol y se los clasificó en tres categorías según su vigor y la presencia de ramas secas: verdes o sanos (árboles con todas las ramas vivas y con buen vigor), parcialmente secos (árboles con una o más ramas primarias secas y vigor reducido) y secos (árboles con todas sus ramas secas) (modificado de Álvarez et al. 2006).

En cada árbol se efectuó una inspección detallada a fin de detectar posibles daños causados por plagas o enfermedades. Se tomaron muestras de los posibles agentes causales de los daños observados. Para la recolección del material biológico se utilizaron lupas de mano, pinzas de punta fina, frascos de vidrio o plástico, líquidos fijadores como alcohol 70\% y solución FAA (formol, acido acético, alcohol). Se registraron los datos en planillas elaboradas para tal fin y mediante fotografías. En el laboratorio, las muestras recolectadas de insectos y otros agentes perjudiciales fueron analizadas e identificadas utilizando microscopios estereoscópicos y binoculares.

En la segunda etapa del proyecto, una vez identificados y analizados los árboles con afecciones, se brindó asesoramiento técnico al personal de la Dirección General de Parques y Paseos de la Municipalidad de la ciudad de Corrientes y se sugirieron medidas de manejo en los casos en que resultó necesario.

La tercera etapa consistió en la realización de un curso-taller teórico práctico destinado a funcionarios y agentes de la mencionada repartición municipal. Se presentaron diversos temas mediante exposiciones teórico- prácticas, con observaciones en laboratorio de insectos y otros agentes causantes de daños, para lo cual se elaboraron guías ilustradas. 


\section{RESULTADOS Y DISCUSIÓN}

Durante los relevamientos en los cuatro espacios verdes analizados fueron censados en total 779 árboles pertenecientes a 67 especies. Los árboles mejor representados por su abundancia fueron: Handroanthus heptaphyllus (lapacho), Peltophorum dubium (ibirá-pitá), Tipuana tipu (tipa), Thuja sp. (tuja), Jacaranda mimosifolia (jacarandá), Delonix regia (chivato), Chorisia insignis (palo borracho), Fraxinus pennsylvanica (fresno), Eugenia uniflora (ñangapirí), Citrus aurantium (naranja agria), Citrus sinensis (naranja dulce), Astronium balansae (urunday), $\mathrm{Li}-$ gustrum lucidum (ligustro), Mangifera indica (mango), Enterolobium contortisiliquum (timbó), Erytbrina crista-galli (ceibo) y Araucaria angustifolia (pino paraná).
La riqueza específica de árboles varió entre 20 (Parque Camba Cuá) y 39 (Parque Mitre) en los espacios verdes considerados (Tabla 1). Con respecto a su estado sanitario, el 85,24\% de los árboles resultaron sanos o verdes y los árboles parcialmente secos y secos se registraron en menores cantidades (Tabla 1).

E1 25,03\% del total de árboles analizados presentaron daños macroscópicos y se reconocieron distintos agentes perjudiciales que afectaron su vitalidad. Los porcentajes de árboles afectados variaron entre el 27,59\% y el 20,91\%, con el mayor de estos valores registrado para el Parque Mitre (Tabla 1).

Entre los factores abióticos que afectaron la salud de los árboles, pudieron reconocerse agentes químicos como pinturas de di-

\begin{tabular}{|l|c|c|c|c|c|c|}
\hline $\begin{array}{l}\text { Espacio } \\
\text { Verde }\end{array}$ & S & $\begin{array}{c}\text { Total de } \\
\text { árboles }\end{array}$ & $\begin{array}{c}\text { Sanos o } \\
\text { verdes }\end{array}$ & $\begin{array}{c}\text { Parcialmente } \\
\text { secos }\end{array}$ & Secos & Afectados \\
\hline $\begin{array}{l}\text { Parque } \\
\text { Mitre }\end{array}$ & 39 & 308 & 233 & 68 & 7 & $85(27,59 \%)$ \\
\hline $\begin{array}{l}\text { Parque } \\
\text { Cambá }\end{array}$ & 20 & 113 & 84 & 29 & 0 & $28(24,78 \%)$ \\
Cua & 27 & 153 & 151 & 2 & 0 & $32(20,91 \%)$ \\
\hline $\begin{array}{l}\text { Plaza } \\
\text { Torrent }\end{array}$ & 23 & 205 & 196 & 8 & 1 & $50(24,39 \%)$ \\
\hline $\begin{array}{l}\text { Plaza de } \\
\text { la Cruz }\end{array}$ & 23 & & & & & \\
\hline
\end{tabular}

Tabla 1: Riqueza específica, número total, estado sanitario y porcentaje de árboles afectados por agentes perjudiciales en espacios verdes de la ciudad de Corrientes.

versos tipos aplicadas sobre troncos y ramas y agentes mecánicos como clavos, alambres, ganchos metálicos, carteles, cables telefónicos y de televisión. Se reconocieron además cicatrices de distinta antigüedad, practicadas con elementos cortantes de diversos tipos.
Los principales síntomas y daños detectados, que se asocian habitualmente con problemas de salud de los árboles (Boa 2008), incluyeron alteraciones en el crecimiento y desarrollo como formación de agallas, deformaciones de troncos y ramas y crecimiento reducido. También se reco- 
nocieron focos de necrosis representadas por manchas, lesiones y cancros. Entre las evidencias físicas de la acción de plagas bióticas se observaron daños por alimentación y nidificaciones de insectos, infecciones de hongos y exudados. Además, fueron identificados crecimientos de otras especies vegetales hemiparásitas y epífitas sobre árboles.

Los principales agentes biológicos perjudiciales al arbolado en los parques y plazas analizados resultaron los hongos de la madera, hormigas, termitas y pulgones. Los porcentajes de hallazgos de árboles infestados en cada sitio, con los cuatro grupos principales de agentes perjudiciales, se presentan en la Figura 1. Las hormigas resultaron el grupo de insectos de mayor abundancia (52,11\% de los hallazgos en 111 árboles) y se localizaron en los cuatro espacios verdes, como también los hongos de la madera (61 árboles atacados). Las termitas fueron detectadas en 28 árboles del Parque Mitre y en un ejemplar de la Plaza Torrent, en tanto que los pulgones infestaron 20 árboles en la Plaza de la Cruz y dos en el Parque Mitre.

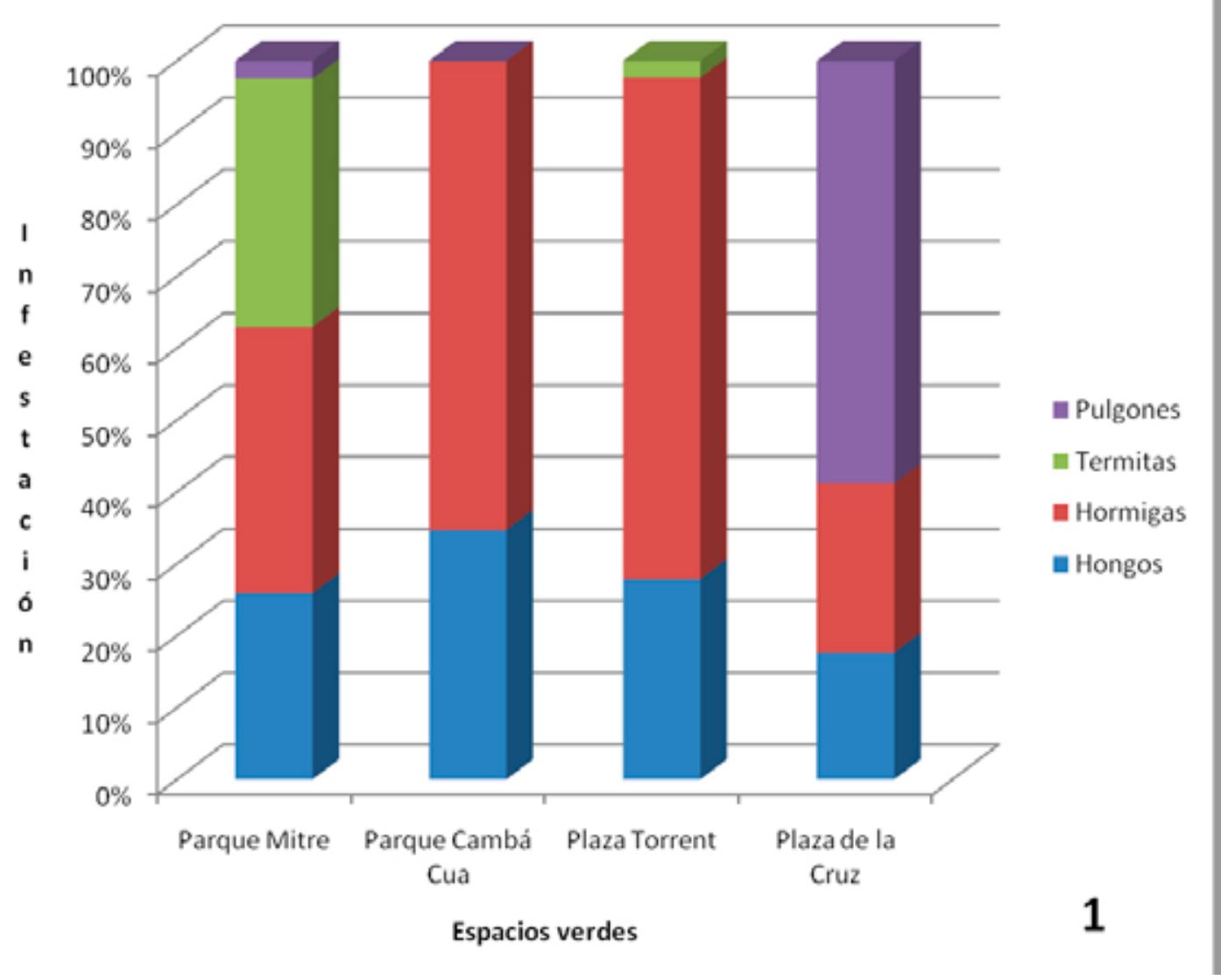

Figura 1: Árboles infestados en cada espacio verde con los cuatro grupos principales de agentes perjudiciales detectados. 
Con respecto a las características biológicas de los organismos perjudiciales, es posible señalar que las hormigas (Hymenoptera, Formicidae) más frecuentes en los parques y plazas de Corrientes se conocen comúnmente como hormigas carpinteras (Camponotus spp.) y hormigas podadoras o cortadoras (Acromyrmex spp.). Las hormigas carpinteras, reconocibles por su abdomen pardo amarillento de aspecto aterciopelado, realizan nidos excavados en la madera de árboles (Fig. 2) o construcciones urbanas, deteriorándola sin alimentarse de ella ya que su dieta está basada en sustancias azucaradas (Medan \& Josens 2005). Por su parte, las hormigas cortadoras presentan espinas en el dorso del tórax y trasladan trozos de hojas y ramas al interior de sus nidos subterráneos donde las utilizan como sustrato para cultivar el hongo del cual se alimentan. Este grupo constituye una de las principales plagas en sistemas agrosilviculturales de la región Neotropical (Della Lucia 2003).
Los hongos de la madera, presentes también en los cuatro sitios de muestreo, producen enfermedades conocidas como pudrición blanca y pudrición castaña de la madera. En ambos casos, ésta pierde gradualmente sus propiedades normales $y$ toma consistencia esponjosa, filamentosa o laminada y se observa manchada y descolorida (Schmidt \& Czeschlik 2006).

Las termitas (Isoptera, Termitidae) causantes de las infestaciones observadas pertenecen a la especie Nasutitermes corniger que se alimenta de madera de árboles vivos y muertos sobre los que nidifica y de madera utilizada en edificaciones. Esta termita es considerada actualmente como una de las especies invasoras más perjudiciales, con una amplia distribución en la Región Neotropical que abarca desde el sur de México al norte de Argentina, con introducciones en Florida (USA) (Scheffrahn et al. 2005). En la ciudad de Corrientes se ha detectado

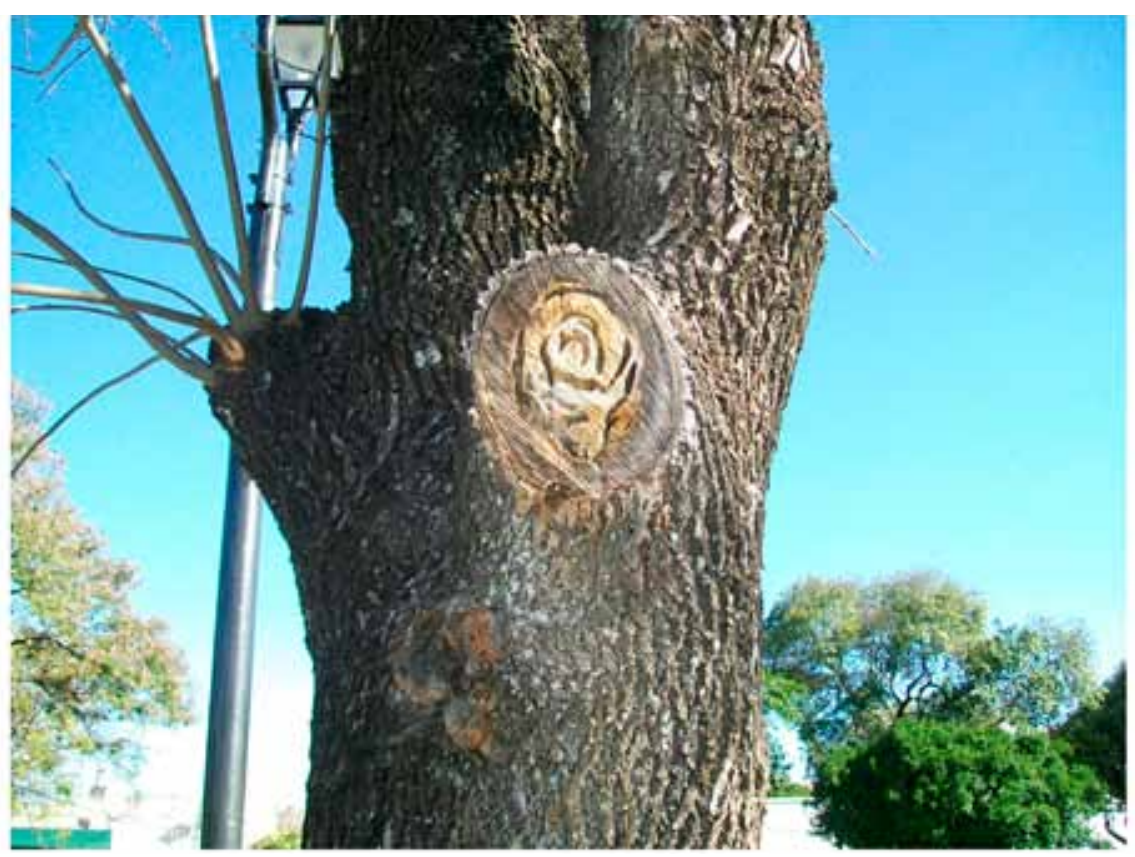

Figura 2: Árbol con excavaciones en tronco y ramas, ocasionadas por hormigas carpinteras del género Camponotus. Parque Cambá Cuá (Corrientes). 
su presencia en árboles y construcciones de barrios vecinos a estos espacios verdes (Torales et al. 1990, Torales 2002).

Los daños ocasionados por pulgones (Hemiptera, Aphididae) se evidencian en el follaje de los árboles, ya que las colonias de estos insectos se localizan principalmente en el envés de las hojas y en brotes, que sufren deformaciones, retardos en su crecimiento y marchitez. Las colonias de algunos pulgones son atendidas por distintos géneros de hormigas, entre ellos Camponotus (Szpeiner 2008).

Entre los organismos que crecen sobre árboles se pudo registrar a plantas hemiparásitas (Phoradendron liga) y epífitas ( $T i-$ llandsia spp. o clavel del aire) que si bien no causan daño directo, pueden ocasionar daños de menor magnitud como caída de ramas por peso excesivo (Boa 2008).

En base a los resultados de los releva- mientos se llevó a cabo la segunda etapa del proyecto, que consistió en la elaboración e implementación de medidas de manejo y prevención de infestaciones en aquellos árboles donde se evidenciaron daños de mayor magnitud. Las mismas se refirieron principalmente a la realización de podas adecuadas en tiempo y forma, a la aplicación de productos fitosanitarios apropiados a cada plaga detectada y a la extracción de árboles muertos y tocones así como de nidos arbóreos de termitas. Se hizo hincapié en la necesidad de detectar prontamente y manejar los insectos perjudiciales presentes ya que los árboles de los espacios verdes actúan como reservorio de los mismos, desde donde se difunde la infestación a edificaciones y árboles cercanos. En esta etapa se trabajó conjuntamente con el personal de la Dirección de Parques y Paseos.

La tercera etapa del Proyecto se cumplió mediante la realización del Curso-Taller "Plagas y enfermedades del arbolado urbano

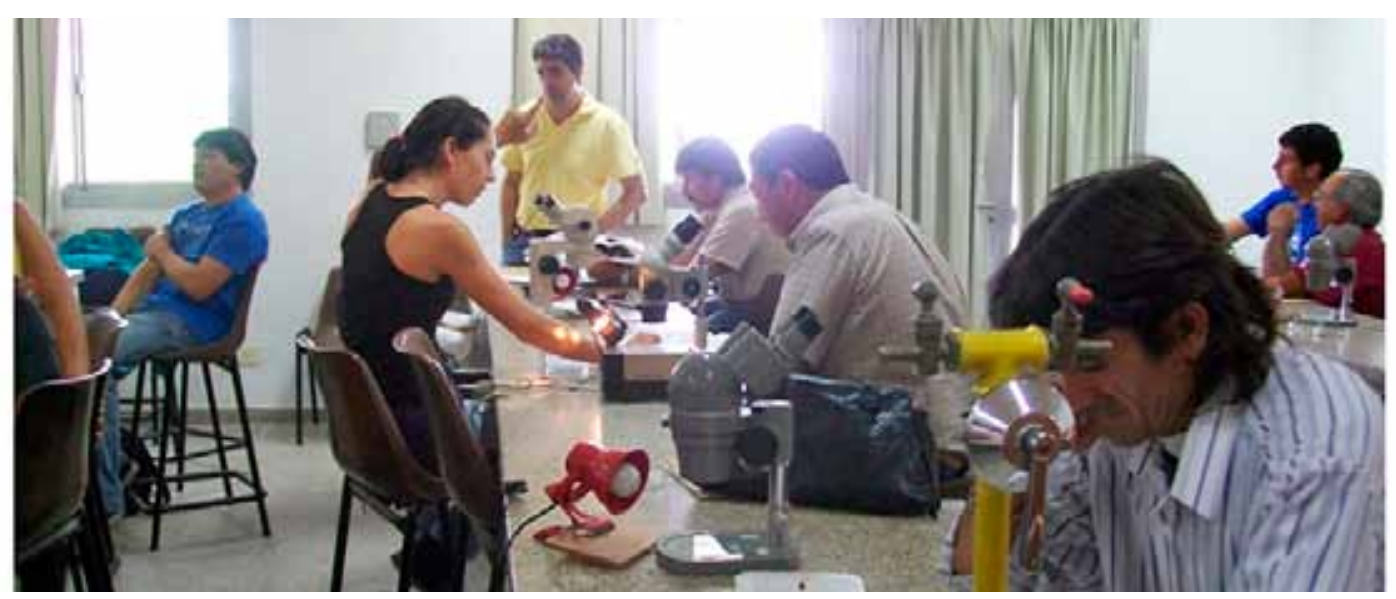

Figura 3: Actividades del curso- taller realizado en la Facultad de Cs. Exactas y Naturales y Agrimensura (UNNE).

de la Ciudad de Corrientes" del cual participaron 21 funcionarios y agentes de la $\mathrm{Di}^{-}$ rección General de Parques y Paseos de la Municipalidad de la Ciudad de Corrientes.
Esta actividad fue desarrollada en aulas y laboratorios de FaCENA y consistió en establecer un espacio de intercambio entre docentes, estudiantes y personal municipal. 
Entre los temas abordados se destacaron aquellos referidos a: importancia ambiental del arbolado urbano, identificación y/o diagnóstico de los principales agentes perjudiciales en Corrientes, consecuencias de los ataques y medidas de manejo. Las exposiciones y trabajos de laboratorio estuvieron a cargo tanto de los docentes como de los estudiantes integrantes del proyecto, quienes participaron activamente y mostraron un excelente desempeño (Fig. 3). Para las observaciones del material biológico, se utilizó una guía ilustrada de reconocimiento e identificación de las principales plagas y enfermedades encontradas durante los muestreos, elaboradas especialmente por el equipo docente del Proyecto. Se destacaron los valiosos aportes y comentarios del personal municipal, que resultaron de gran interés dada la amplia experiencia en la temática de varios de los agentes asistentes.

\section{BIBLIOGRAFÍA}

Alvarez, J.A.; P. Villagra; M.A. Cony; E.M. Cesca \& J.A. Boninsegna. 2006. Estructura y estado de conservación de los bosques de Prosopis flexuosa D.C. (Fabaceae, subfamilia: Mimosoideae) en el noreste de Mendoza (Argentina). Rev. Chil. Hist. Nat. 79: 75-87.

Boa, E. 2008. Guía ilustrada sobre el estado de salud de los árboles: Reconocimiento e interpretación de síntomas y daños. San Salvador: FAO- OIRSA.

Buduba, C. 2004. Arbolado urbano patagónico: algunos conceptos para su defensa y cuidado. Patagonia Forestal 10 (4): 11- 14.

Cian, D.; C. Passicot \& E. Cabral. 2000. Guía práctica para reconocer las plantas del Parque Mitre, para docentes y alumnos de

\section{CONCLUSION}

Los agentes perjudiciales al arbolado urbano muestran particularidades propias en cada ciudad. Por ello, las actividades desarrolladas en el marco de este proyecto de extensión permitieron contar con datos precisos del estado sanitario de los árboles de espacios verdes representativos de la ciudad de Corrientes al momento de este estudio e identificar los principales problemas que los afectan a fin de mejorar las estrategias de prevención y manejo. Asimismo, resultó destacable la interacción con el personal municipal que sentó las bases para continuar este tipo de actividades y representó un valioso espacio de trabajo conjunto.

la EGB 3 y Polimodal. Comunicaciones Científicas y Tecnológicas UNNE. (http:// www.unne.edu.ar/unnevieja/Web/cyt/

$$
\text { cyt/2000/cyt.htm) }
$$

Comerci, R.E. 2010. Catálogo de plagas y enfermedades del arbolado urbano, descripción, control y tratamientos. Buenos Aires: Orientación Gráfica.

Della Lucia, T.M.C. 2003. Hormigas de importancia económica en la región Neotropical (pp. 337- 349). En: Fernández,F. (ed.), Introducción a las hormigas de la región Neotropical. Bogotá: Instituto Humboldt.

Fiorentino,D.\&L.Diodato.1991.Breve panorama de las plagas entomológicas forestales argentinas. Investigación Agraria 1: 181- 190. 
Fontes, L.R. 1995. Cupins en áreas urbanas (pp. 57- 76). En: Berti Filho, E \& L.R. Fontes (eds.), Aspectos atuais da biología e controle de cupins. Piracicaba: FEALQ.

Fontes, L.R. 1998. Consideraçôes sobre a complexidade da interação entre o cupim subterrâneo Coptotermes havilandai e a arborização no ambiente urbano (pp. 109-124). En: Fontes, L.R. \& E. Berti Filho (eds.), Cupins, o desafio do conbecimento. Piracicaba: FEALQ.

Galdeano, E.; L.E. Torres; N. Meneguzzi; F. Guzmán; G. Gomez; D. Docampo \& L. Conci. 2004. Molecular Characterization of $16 S$ Ribosomal DNA and Phylogenetic Analysis of Two $X$-disease Group Phytoplasmas Affecting China-tree (Melia azedarach L.) and Garlic (Allium sativumL.) in Argentina. J. Phytopathol. 152: 174- 181.

Gómez Lopera, F. 2005. Las zonas verdes como factor de calidad de vida en las ciudades. Ciudad y Territorio 37(144): 417-436.

Kopta, R.G.; R.F. Kopta \& M.H. Ezquerro. 1997. Manual de poda del arbolado público. Otras sugerencias para mejorar las forestaciones urbanas. Córdoba, Argentina: Fundación Ambiente, Cultura y Desarrollo.

Medan, V. \& R.B. Josens. 2005. Nectar foraging behaviour is affected by ant body size in Camponotus mus. J. Insect Physiol. 51: 853- 860.

Milano, S. \& L.R. Fontes. 2002. Cupim e Cidade: implicações ecológicas e controle. São Paulo.

Scheffrahn, R.H.; J. Krecek; A.L. Szalanski \& J.W. Austin. 2005. Synonymy of Neotropical arboreal termites Nasutitermes corniger and $N$. costalis (Isoptera: Termitidae: $N a-$ sutitermitinae), with evidence from morphology, genetics and biogeography. Ann. Entomol. Soc. Am. 98 (3): 273- 281.

Schmidt, O. \& D. Czeschlik. 2006. Wood and tree fungi: Biology, damage, protection, and use. Berlin: Springer.

Szpeiner, A. 2008. Aphididae (Hemiptera) on ornamental plants in Córdoba (Argentina). Rev. Soc. Entomol. Argent. 67 (1-2): 49-56.

Torales,G.J.;E.B.Oscherov;A.C.Armúa;M.M. Martegani; E.R. Laffont \& M.O. Arbino. 1990. Contribución al conocimiento de las termitas de Argentina (Provincia de Corrientes). Nasutitermes corniger Motschulsky (Isoptera: Termitidae, Nasutitermitinae). II Parte. Infestación de viviendas. Facena 8: 9- 25.

Torales, G.J. 2002. Termitas as structural pest in Argentina. Sociobiology 40 (1): 191- 206. 